\title{
On Chip Protein Pre-Concentration for Enhancing the Sensitivity of Porous Silicon Biosensors
}

\author{
Sofia Arshavsky-Graham, ${ }^{\dagger, \dagger}$ Naama Massad-Ivanir, ${ }^{\dagger}$ Federico Paratore, $^{\S}, \|$ Thomas Scheper, \\ Moran Bercovici, ${ }^{\S, \perp}{ }^{(1)}$ and Ester Segal ${ }^{*},+, \perp_{(0)}$ \\ ${ }^{\dagger}$ Department of Biotechnology and Food Engineering, ${ }^{\S}$ Department of Mechanical Engineering, and ${ }^{\perp}$ The Russell Berrie \\ Nanotechnology Institute, Technion - Israel Institute of Technology, Haifa 3200003, Israel \\ ${ }^{\ddagger}$ Institute of Technical Chemistry, Leibniz Universität Hannover, Callinstr. 5, 30167 Hanover, Germany \\ "IBM Research - Zürich, Säumerstrasse 4, 8803 Rüschlikon, Switzerland \\ Supporting Information
}

ABSTRACT: Porous silicon (PSi) nanomaterials have been widely studied as label-free optical biosensors for protein detection. However, these biosensors' performance, specifically in terms of their sensitivity (which is typically in the micromolar range), is insufficient for many applications. Herein, we present a proof-of-concept application of the electrokinetic isotachophoresis (ITP) technique for real-time preconcentration of a target protein on a PSi biosensor. With ITP, a highly concentrated target zone is delivered to the sensing area, where the protein target is captured by immobilized aptamers. The detection of the binding events is conducted in a label-free manner by reflective interfero-

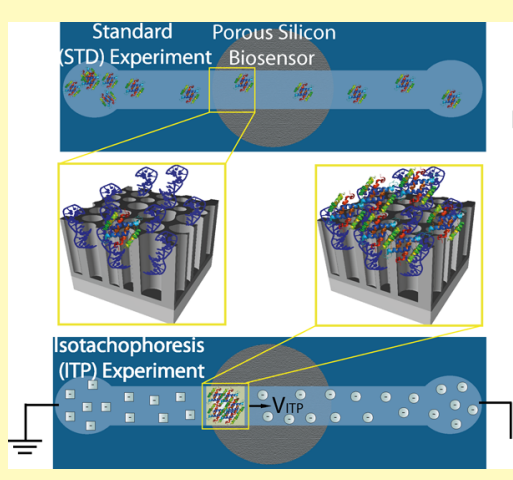
Leading His-tag Binding
Electrolyte lon Aptamer
Terminating His-tagged
Electrolyte lon Protein metric Fourier transformation spectroscopy (RIFTS). Up to 1000-fold enhancement in local concentration of the protein target and the biosensor's sensitivity are achieved, with a measured limit of detection of $7.5 \mathrm{nM}$. Furthermore, the assay is successfully performed in complex media, such as bacteria lysate samples, while the selectivity of the biosensor is retained. The presented assay could be further utilized for other protein targets, and to promote the development of clinically useful PSi biosensors.

KEYWORDS: porous silicon, optical biosensor, isotachophoresis, aptamer, label-free

$S$ ince the seminal work of Sailor and co-workers in 1997,' porous silicon ( $\mathrm{PSi}$ ) nanomaterials have been extensively studied as optical transducers in a variety of biosensing schemes. $^{2-5}$ Different PSi-based optical structures, such as Fabry-Pérot thin films, ${ }^{1,6}$ Bragg mirrors, ${ }^{7}$ rugate filters, ${ }^{8,9}$ and microcavities, ${ }^{10,11}$ have been exploited for detection of small molecules, ${ }^{12-15}$ DNA, ${ }^{1,16-18}$ proteins, ${ }^{19,20}$ enzymes, ${ }^{21,22}$ and whole cells. $^{23-26}$ In these biosensors, selective capture of a target analyte within the PSi nanostructure, by surfaceimmobilized receptors, induces measurable shifts in the reflectivity spectrum ${ }^{1,27,28}$ allowing for real-time and label-free detection and quantification of the target in a simple yet reliable manner. ${ }^{1,20,27-29}$ Despite the significant advantages of PSi biosensors, their application is limited due to their relatively low sensitivity, attributed mainly to the hindered diffusion of analytes into the porous matrix. ${ }^{4,30-33}$ Thus, most of the reports to date present measurements in the micromolar range for both $\mathrm{DNA}^{17,34}$ and protein ${ }^{3,19,20,35-37}$ targets.

Significant research efforts have been directed toward improving the sensitivity of PSi-based biosensors. Several label-free strategies have been proposed for enhancing the sensitivity. Recently, Barillaro and co-workers presented a new signal processing technique which relies on the calculation of the average value spectral interferograms over wavelength, demonstrating detection of $150 \mathrm{pM}$ bovine serum albumin (BSA) by nonspecific adsorption in a PSi Fabry-Pérot thin film. ${ }^{38}$ In a later study, this methodology was applied for the detection of a protein biomarker of inflammation and sepsis at a concentration as low as $3 \mathrm{nM}^{39}$ Weiss and co-workers developed a PSi microcavity membrane for a flowthrough biosensing platform, which improves the analyte transport into the pores and enables the detection of $500 \mathrm{nM}$ streptavidin within 20 min. $^{31}$

Several recent studies have demonstrated the potential in using isotachophoresis (ITP) focusing to enhance surface-based reactions. ${ }^{40-43}$ ITP is a well-established electrophoretic method that utilizes a discontinuous buffer system for simultaneous extraction, separation, and concentration of ionic species, based on their effective electrophoretic mobility. ${ }^{44-46}$ Yet, as all the reported assays are based on fluorescence detection of the

Received: September 14, 2017

Accepted: November 22, 2017

Published: November 22, 2017 
(a)

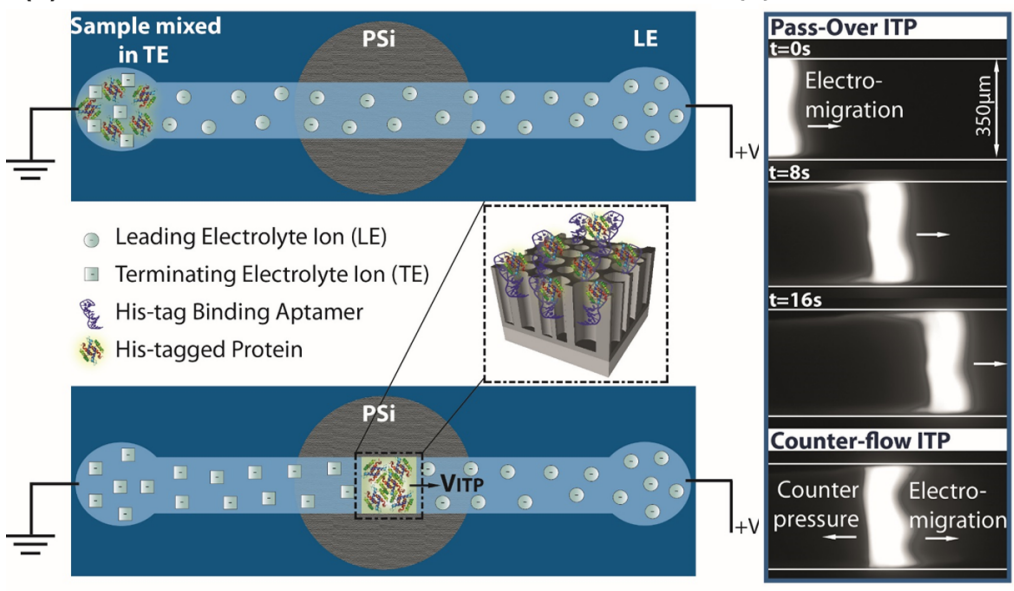

(c)

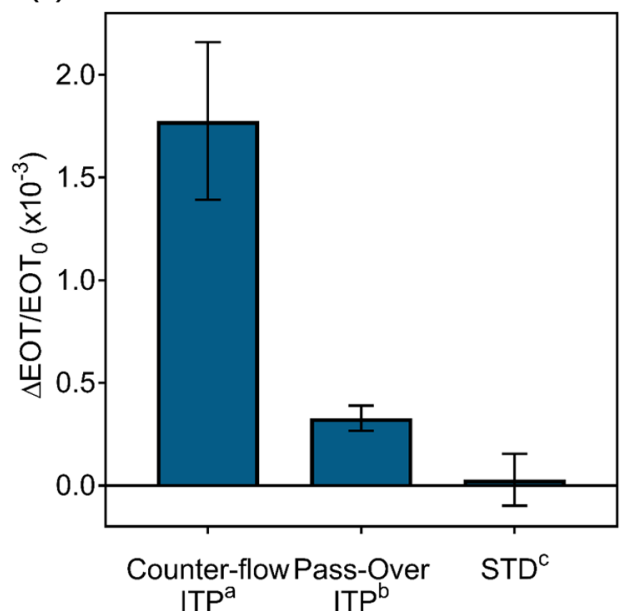

Figure 1. (a) Schematic illustration of the $\mathrm{PSiO}_{2}$ biosensor setup and an ITP-based biosensing experiment. Upper panel: the target protein sample is mixed with TE buffer in the west reservoir, while the channel and the east reservoir are filled with LE buffer. Lower panel: as voltage is applied, the target protein focuses at the LE-TE interface and electromigrates along the channel toward the $\mathrm{PSiO}_{2}$ sensing area where it is captured by the targeting aptamers. (b) Raw fluorescence images of the target protein, focusing under different modes of ITP assay. (c) Averaged relative $\Delta \mathrm{EOT}$ signal for counter-flow ITP, pass-over ITP, and standard (STD) configurations for $750 \mathrm{nM}$ target protein, demonstrating the profound increase in signal upon counter-flow ITP mode ( $n \geq 2$ for each data set). Different superscript letters $\left({ }^{\mathrm{a}, \mathrm{b}, \mathrm{c}}\right)$ indicate a statistically significant difference $(t$ test, $p<$ $0.05)$.

target, they require target labeling or utilization of a labeled secondary capture probe. In recent work, we have demonstrated interfacing the PSi Fabry-Pérot interferometer with ITP to achieve highly sensitive and label-free detection of nucleic acids. ${ }^{30}$ In the present work, we extend the applicability of this platform for a real-time preconcentration of a protein target. As a model biosensing system, we utilize a PSi-based aptasensor for detection of his-tagged proteins, which is a characterized biosensor with a micromolar-range sensitivity. ${ }^{20}$ An oxidized PSi $\left(\mathrm{PSiO}_{2}\right)$ thin film, with an insulating oxide layer, is chemically functionalized with polyhistidine tag (his-tag) binding aptamers, ${ }^{20,47-49}$ and integrated with a microfluidic system. ITP is then applied to focus the target protein into a highly concentrated protein zone and deliver it to the PSi biosensor. By holding this concentrated protein zone stationary on top of the biosensor via counter-flow, ${ }^{30}$ the target protein is able to diffuse into the porous layer and interact with the capturing aptamers. The binding events are monitored throughout the entire ITP process with reflective interferometric Fourier transformation spectroscopy (RIFTS) method. We demonstrate detection of the target protein at concentrations as low as $7.5 \mathrm{nM}$ and up to 1000 -fold enhancement in sensitivity. Importantly, we show that this assay can be successfully applied in complex biological fluids such as bacterial lysate samples.

\section{RESULTS AND DISCUSSION}

A schematic illustration of the biosensor design and corresponding assay is presented in Figure 1a. The biosensor is constructed from a PSi Fabry-Pérot thin film, used as an optical transducer, and aptamers specifically binding the his-tag sequence of proteins ( $6 \mathrm{H} 7$ aptamer) are conjugated to the porous nanostructure. ${ }^{20,47-49}$ This biosensor, previously developed by our group, ${ }^{20}$ is adapted as a model system for demonstrating the applicability of ITP for preconcentration of proteins on PSi. As the behavior of aptamers may vary drastically when conjugated to a surface, ${ }^{50}$ working with a wellcharacterized system is important for proper characterization of the biosensor. The PSi nanostructure is fabricated by anodization of a heavily doped p-type crystalline Si wafer at a constant current density of $375 \mathrm{~mA} \mathrm{~cm}^{-2}$ for $30 \mathrm{~s}$. The resulting porous film is thermally oxidized at $1000{ }^{\circ} \mathrm{C}$ for $46 \mathrm{~h}$ under oxygen flow to create a robust insulating oxide layer that can withstand the high voltage applied during ITP. ${ }^{30}$ Highresolution scanning electron microscopy of the oxidized film reveals that the integrity of the delicate porous nanostructure is maintained during the harsh oxidation step and interconnecting cylindrical pores with an average diameter of $45 \pm 10 \mathrm{~nm}$ are observed (Figure S1, Supporting Information). The porous nanostructure thickness is $4.8 \mu \mathrm{m}$ and a 780 -nm-thick planar $\mathrm{SiO}_{2}$ layer is observed beneath the $\mathrm{PSiO}_{2}$ layer. Figure $\mathrm{S} 1$ also presents the raw reflectivity data and corresponding FFT spectra of the planar and porous $\mathrm{SiO}_{2}$ layers. Following oxidation, subsequent silanization and carbodiimide coupling steps are used to conjugate the amine-terminated $6 \mathrm{H} 7$ aptamers to the porous surface ${ }^{20}$ and successful immobilization is confirmed by RIFTS (Figure S2). The biosensor is integrated with polydimethylsiloxane (PDMS) microchannels, as illustrated in Figure 1a. The PDMS microchannel, $3 \mathrm{~cm}$ in length, $350 \mu \mathrm{m}$ in width and $25 \mu \mathrm{m}$ in depth, is attached to the $\mathrm{PSiO}_{2}$ by plasma activation. It should be noted that the aptamer immobilization step is carried out following the assembly with the microchannel and adjacent to the biosensing experiments.

One of the main considerations in designing an ITP assay is proper choice of a discontinuous buffer system, comprising a leading electrolyte ion (LE) and terminating electrolyte ion (TE), having higher and lower electrophoretic mobility than the target, respectively. ${ }^{51}$ The buffer system should be chosen according to the charge of the target and optimized to allow maximal ITP focusing, proper functionality of the biological capture probe, and suitability to the $\mathrm{PSiO}_{2}$ substrate, which may be affected by buffers with a high $\mathrm{pH}$ value. ${ }^{43}$ In the present work, the target is a $24 \mathrm{kDa}$ his-tagged protein from the arabinanase family. This protein possesses a theoretical isoelectric point of 5.45 (calculated based on its amino acid sequence) and is accordingly characterized by a net negative 

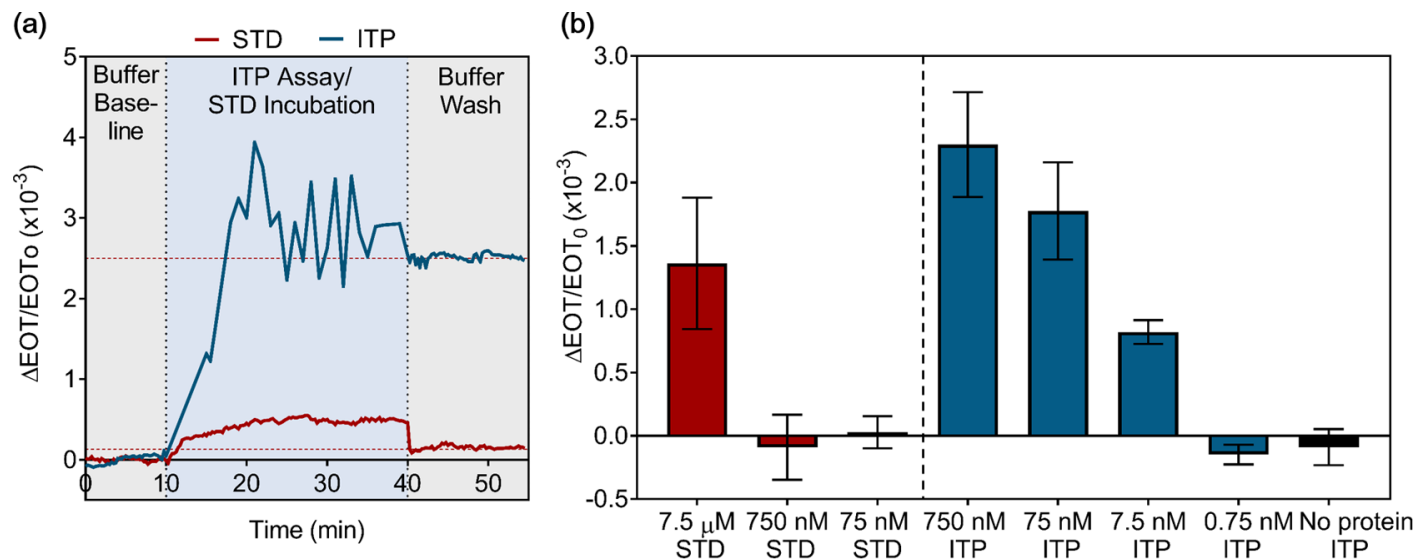

Figure 2. (a) Relative $\triangle \mathrm{EOT}$ changes vs time of the constructed biosensor during typical ITP-based (blue line) and standard (STD) (red line) biosensing experiments with $750 \mathrm{nM}$ target protein. (b) Averaged relative $\triangle \mathrm{EOT}$ signal for different concentrations of the target protein under STD and ITP conditions. Biosensing experiments are also conducted without target protein as a negative control $(n \geq 3$ for each concentration).

charge at a neutral $\mathrm{pH}$. Thus, an anionic ITP is applied and LE and TE buffers are chosen accordingly. $\mathrm{Cl}^{-}$was used as a leading ion, Tricine as a terminating ion and Bis-Tris as the counterion. This buffer system was recently demonstrated for anionic protein focusing with ITP. ${ }^{43}$ In addition, proper function of the aptamers as capture probes is dependent on their correct $3 \mathrm{D}$ folding and typically requires performing the binding reactions in the buffer used in their selection process (i.e., selection buffer). ${ }^{19,20,26,50}$ Herein, the $\mathrm{pH}$ value of the chosen buffer system $(\sim 7)$ resembles that of the $6 \mathrm{H} 7$ aptamer's selection buffer and the LE buffer is further supplemented with $0.15 \mathrm{M} \mathrm{NaCl}$. The latter is not only ideal for the aptamerprotein interaction, but also improves the ITP focusing ratio. ${ }^{43}$ Furthermore, prior to ITP, the selection buffer is introduced into the microchannel to ensure the proper folding and functionality of the immobilized aptamer. As presented in Figure 1a, upper panel, in a typical ITP-based biosensing experiment, the microchannel and the East reservoir are first filled with LE buffer, while the West reservoir is filled with the target protein, mixed in TE buffer. Voltage is then applied along the channel and a sharp electric field gradient is formed at the LE-TE interface where the target protein focuses. The target continuously accumulates at the LE-TE interface and a highly concentrated protein zone is formed. This zone, referred to as the ITP peak, electromigrates along the channel toward the $\mathrm{PSiO}_{2}$ biosensor region where the target protein is captured by the $6 \mathrm{H} 7$ aptamers (Figure 1a, lower panel). At this stage, two different ITP assay modes, pass-over and counter-flow, are tested, presented in Figure $1 \mathrm{~b}$ as raw fluorescence images of the target protein ITP peak. In the pass-over ITP mode, the ITP peak passes over the $\mathrm{PSiO}_{2}$ biosensor without stopping. The interaction of the ITP peak with the sensing area is minimal and only a few seconds in time. In the counter-flow ITP mode, as the ITP peak arrives to the biosensor, it is held stationary for up to $\sim 25 \mathrm{~min}$, by applying a pressure driven flow, which counters the electromigration. Target infiltration into the porous layer and capture by the $6 \mathrm{H} 7$ aptamers are monitored in real-time during the ITP assay by RIFTS, and changes in the effective optical thickness (EOT), which is the product of the average refractive index and the thickness of the porous layer, are computed. It should be noted that the protein is fluorescently labeled in the assay only to allow visualization of the ITP peak location, but no labeling is required for the biosensing experiments. Although not tested herein, the presence of the ITP peak on top of the biosensor could be also controlled by real-time analysis of the EOT signal. Figure 1c summarizes the relative $\triangle \mathrm{EOT}$ changes for biosensing experiments of $750 \mathrm{nM}$ target protein under counter-flow and pass-over ITP modes. For comparison, the obtained signal for a similar experiment using a standard (no ITP) biosensing experiment is also presented. While no change in the EOT signal is observed under standard conditions, a significant EOT increase is obtained when ITP is applied, with both ITP assay modes. Furthermore, a $\sim 5.4$-fold signal enhancement is observed when counter-flow ITP mode is applied, in comparison to the pass-over mode. This is ascribed to the enhanced diffusion flux of the target protein into the porous nanostructure as well as sufficient interaction time to allow binding of the protein to the immobilized aptamers in the counter-flow assay. ${ }^{43}$ Thus, the counter-flow mode is further utilized in this work as will be described next.

Figure 2a compares relative $\triangle \mathrm{EOT}$ changes during time for typical ITP-based and standard biosensing experiments of 750 $\mathrm{nM}$ target protein. First, an EOT baseline is acquired in LE buffer for ITP-based experiment, and a mixture of 2:1 LE and TE, respectively, for standard experiment. This buffer mixture is utilized to simulate the buffer conditions in the ITP peak. Then, for the ITP-based experiment, a $350 \mathrm{~V}$ voltage is applied and ITP is initiated. As the concentrated protein zone arrives to the $\mathrm{PSiO}_{2}$ biosensor region, it is held stationary and the reflectivity spectra are recorded. For the standard experiment, the protein sample is introduced and incubated in the channel for $30 \mathrm{~min}$. It should be noted that the reflectivity spectra in these experiments are recorded under static conditions, with no flow. For both assays a gradual EOT increase is observed until saturation is attained. This increase is attributed to the infiltration of the protein molecules into the pores and binding to the immobilized aptamer. The noisy signal during this step stems from the basic experimental setup (pressure driven flow produced by a water column) used for controlling the location of the ITP focusing zone over the $\mathrm{PSiO}_{2}$ biosensor region and the concurrent reflectivity measurements. Since the stabilization is manual, slight movements of the ITP plug occur during the reflectivity measurements, which result in the observed fluctuations in the signal during this step. Next, the microchannel is thoroughly washed with the baseline buffers and a rapid decrease in the EOT signal is observed for both experiments, as unbound proteins are washed away. Accord- 
(a)

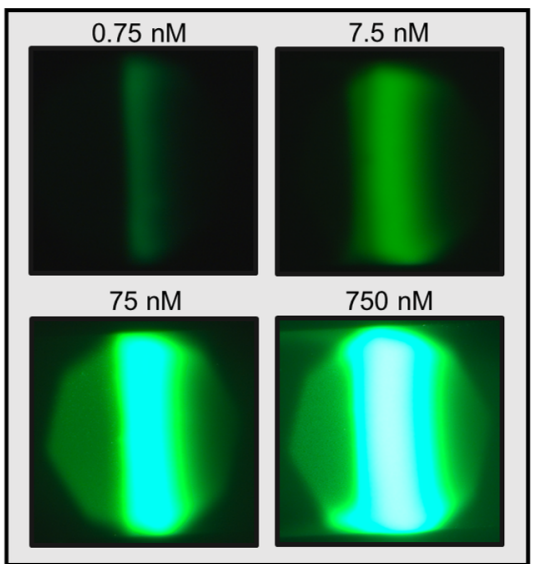

(b)

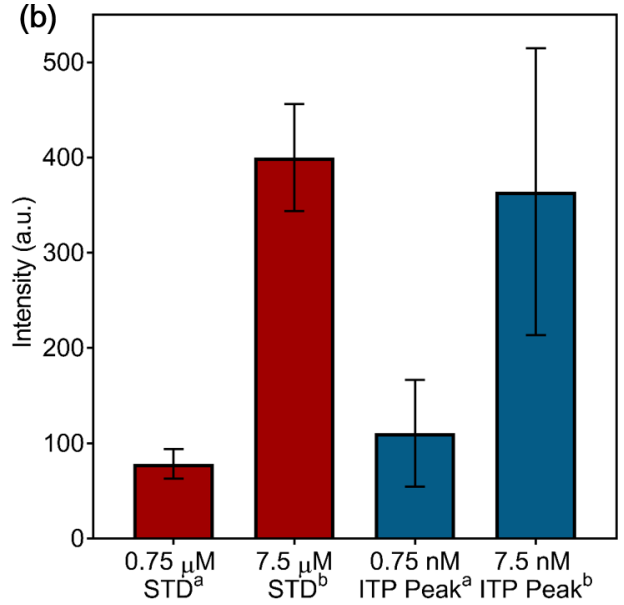

Figure 3. (a) Raw fluorescence images of the ITP peak for different concentrations of the labeled target protein. (b) Comparison of the fluorescence intensity values of the labeled protein measured during standard (STD) vs ITP biosensing experiments $(n \geq 3$ for each data set). Different superscript letters $(\mathrm{a}, \mathrm{b})$ indicate a statistically significant difference $(t$ test, $p<0.05)$.

ingly, this steady state EOT shift is ascribed to the surfacebound targets and constitutes the relevant readout signal of the assay. The attained relative $\triangle \mathrm{EOT}$ value in the ITP-based assay is $2.8 \times 10^{-3}$ (corresponding a net EOT shift of $41 \mathrm{~nm}$ ) in comparison to only $0.15 \times 10^{-3}$ (corresponding a net EOT shift of $2.5 \mathrm{~nm}$ ) in the standard mode, demonstrating a significant signal improvement by ITP. Qualitatively, the change in the EOT as a function of time observed during ITP resembles that of a standard biosensing experiment in which the concentration target protein is $7.5 \mu \mathrm{M}$ (see Figure S3).

Sensitivity enhancement and improvement in LoD are studied by exposure of the biosensor to different concentrations of the target protein $(0.75 \mathrm{nM}-7.5 \mu \mathrm{M})$ under ITP and standard configurations. The results are summarized in Figure $2 \mathrm{~b}$, presenting the averaged relative $\triangle \mathrm{EOT}$ changes for each concentration (summarizing $n \geq 3$ repeats for each). For standard conditions, an EOT signal is only observed for protein concentrations $\geq 7.5 \mu \mathrm{M}$, while for lower concentrations of 750 $\mathrm{nM}$ and $75 \mathrm{nM}$ no EOT shifts are detected. These results are in agreement with the micromolar detection range observed by Urmann et al. ${ }^{20}$ When applying ITP, EOT changes are already detected at a target concentration of $7.5 \mathrm{nM}$, demonstrating a significant enhancement in sensitivity of the biosensor and an improvement in the LoD by more than 100-fold and up to 1000 -fold (a typical ITP experiment with $7.5 \mathrm{nM}$ target is presented in Figure S4, and raw reflectivity data respective FFT spectra are depicted in Figure S5). It should be pointed out that the dissociation constant of the $6 \mathrm{H} 7$ aptamer is only $4.6 \mu \mathrm{M}^{47}$ Thus, the presented assay allows overcoming the limited binding affinity of the capture probe by detection of the protein with initial concentration far below the dissociation constant. In standard experiments, the target delivery to the biosensor is governed by diffusion and reaction kinetics. For protein concentrations of 750 and $75 \mathrm{nM}$ (which are below the $K_{d}$ of the aptamer), the reaction kinetics is limited by the reaction offrate, which dominates this concentration regime and is characterized by single-molecule binding and debinding events. ${ }^{52}$ ITP, which increases the local target concentration above the biosensor, affects both diffusion and reaction kinetics. The former is accelerated due to the higher concentration gradient, while the latter is enhanced by pushing the reaction to a regime dominated by the reaction on-rate. Additional contribution of counter-flow ITP assay is by introducing sample mixing in the ITP plug, ${ }^{53}$ which further enhances the target flux to the biosensor. The poor performance of ITP at a protein concentration of $0.75 \mathrm{nM}$ may be attributed to dispersion, which arises from electroosmotic flow (EOF). Note that no EOF suppressing agents, such as polyvinylpyrrolidone, were used in the present study, as our previous work has shown that the polymer may affect the integrity of the optical readout. ${ }^{30}$ Yet, effects ascribed to reduction in fluorescence signal (see Figure 3a) possibly due to fluorophore adsorption to the PDMS microchannel and photobleaching may be also considered as they result in loss of peak visualization and consequently significant reduction in interaction time (to $\sim 10$ $\mathrm{min}$, in comparison to $\sim 25 \mathrm{~min}$ for higher protein concentrations). As the ITP assay involves a complex buffer system, the biosensor response to the buffers (no target protein) is also characterized. Figure S6 presents the relative $\triangle \mathrm{EOT}$ changes during time for these experiments, where the electromigration of the LE-TE interface is monitored by realtime analysis of the EOT changes and exploits the significant difference in refractive index between LE and TE buffers. No EOT changes are detected during these experiments (Figure $2 \mathrm{~b})$, confirming that the optical signal is only ascribed to binding events occurring within the porous nanostructure.

The actual target protein concentration within the ITP peak is estimated by analysis of the target fluorescence intensity. Figure 3a presents raw fluorescence images of the ITP peak of different concentrations of the target protein. The analysis of the fluorescence signal is conducted for the lower concentration range (Figure 3a, upper panel) due to signal saturation for protein concentrations of $750 \mathrm{nM}$ and $75 \mathrm{nM}$ (Figure 3a, lower panel). Figure $3 \mathrm{~b}$ compares the maximal fluorescence intensity of the ITP peak for target concentrations of $7.5 \mathrm{nM}$ and 0.75 $\mathrm{nM}$ to the fluorescence values observed under standard experiment with the protein at $7.5 \mu \mathrm{M}$ and $0.75 \mu \mathrm{M}$. No significant difference ( $t$ test, $p>0.05)$ in fluorescence intensity of the target protein is observed between $7.5 \mathrm{nM}$ ITP peak and $7.5 \mu \mathrm{M}$ standard experiment, and $0.75 \mathrm{nM}$ ITP peak and 0.75 $\mu \mathrm{M}$ standard experiment. These results suggest a 1000-fold increase in the local target concentration within the ITP peak, compared to the initial concentration of the sample, for the corresponding concentrations. Furthermore, for $0.75 \mathrm{nM}$ target protein, the actual concentration within the ITP peak is 

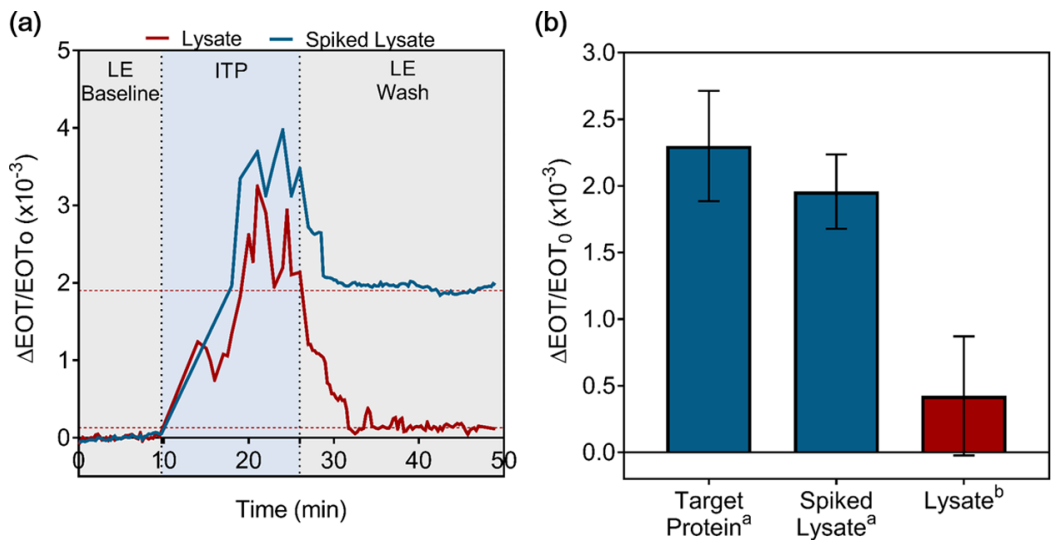

Figure 4. (a) Relative $\triangle E O T$ changes vs time of the aptamer-based biosensor during a typical ITP experiment with E. coli lysate suspension (with protein concentration of $20 \mu \mathrm{g} \mathrm{mL}^{-1}$ ) spiked or nonspiked with $18 \mu \mathrm{g} \mathrm{mL}^{-1}$ of the target protein (corresponding to $750 \mathrm{nM}$ ). (b) Averaged relative $\triangle \mathrm{EOT}$ signal for ITP biosensing experiments of neat target protein, E. coli lysate spiked with the target protein, and neat $E$. coli lysate (no target protein), demonstrating great performance and selectivity of the assay in a highly complex media $(n=4$ for each data set). Note: for neat lysate samples, a fluorescently labeled lysate is utilized, while for spiked samples, a nonlabeled lysate is used. Different superscript letters (a,b) indicate a statistically significant difference $(t$ test, $p<0.05)$.

estimated to be $0.75 \mu \mathrm{M}$, far below the dissociation constant of the $6 \mathrm{H} 7$ aptamer, suggesting that for protein's concentrations lower than $7.5 \mathrm{nM}$, the sensitivity is limited by the aptamer's affinity and not by the ITP assay. Thus, by utilizing different capture probes with lower dissociation constants, higher sensitivity and lower LoD may be achieved.

The selectivity of the biosensor under ITP and its performance in complex media, rich with proteins, are studied by introduction of $E$. coli lysate suspension (with protein concentration of $20 \mu \mathrm{g} \mathrm{mL}^{-1}$ ) spiked with $18 \mu \mathrm{g} \mathrm{mL}^{-1}$ of the target protein (corresponding to $750 \mathrm{nM}$ ). This complex sample is rich in nontarget proteins, with different molecular weights, as presented in the SDS-PAGE analysis in Figure S7. Figure $4 \mathrm{a}$ depicts the relative $\triangle \mathrm{EOT}$ changes vs time in this set of experiments in comparison to behavior of a neat lysate (no target protein). For both experiments, a gradual increase in the EOT signal is observed, as the target and nontarget proteins infiltrate into the porous layer. However, upon a washing step with LE buffer, as the nontarget proteins are washed away, the EOT signal rapidly decreases for lysate sample to its original EOT value, while for the spiked lysate sample, a profound relative $\triangle \mathrm{EOT}$ signal of $1.94 \times 10^{-3}$ (corresponding to a net $\Delta$ EOT value of $33 \mathrm{~nm}$ ) is observed. This signal is attributed to the selective binding of the target protein by the $6 \mathrm{H} 7$ aptamers from a diverse concentrated mixture of nontarget proteins. Figure $4 \mathrm{~b}$ compares the averaged relative $\triangle \mathrm{EOT}$ changes for neat target protein, E. coli lysate spiked with the target protein and neat lysate (no target protein) upon ITP biosensing experiments. A similar EOT signal $(t$ test, $p>0.05)$ is obtained for a neat target protein and the target protein within the lysate sample, demonstrating the excellent performance of the constructed biosensor in detecting the target protein upon ITP, even in a highly complex sample overabundant with nontarget proteins and shorter interaction time. The ITP peak in the presence of the lysate sample, overloaded with a diversity of proteins, could not be held stably stationary above the sensing area, resulting in reduction of interaction time to $\sim 10$ min, in comparison to $\sim 25$ min with a neat protein sample. This may be ascribed to dispersion due to EOF. Additionally, this suggests that reduction in assay time without significantly affecting the EOT signal may be possible. For neat lysate samples, a significantly lower EOT signal $(t$ test, $p<0.05)$ is observed, attributed to a minimal nonspecific adsorption of nontarget proteins. Additional feature of ITP is its ability to separate the ionic species within the sample, based on their effective electrophoretic mobility. This is demonstrated in Figure S8, presenting the sample separation by ITP of a targetspiked lysate. Although not investigated extensively herein, this feature has the potential to further improve the specificity of the biosensor, separating the target from other interfering species and creating a neat target plug. This is especially valuable when analyzing complex biological fluids, loaded with nontarget molecules.

In this proof-of-concept work we have demonstrated the applicability of ITP for protein focusing on PSi biosensor. Utilizing ITP, we were able to enhance the local target protein concentration at the sensing area by 1000 -fold and maximize the optical signal. A nanomolar LoD is demonstrated in less than $50 \mathrm{~min}$ assay time. Furthermore, the successful performance in highly complex bacteria lysate samples demonstrates the tremendous potential of the assay for constructing clinically relevant biosensing schemes. This is the first time that ITP has been applied for protein focusing on PSi biosensors, as well as the utilization of immobilized aptamers as capture probes in an ITP assay. This assay is independent of other signal amplification techniques and could be combined with other strategies to further enhance the sensitivity of PSi biosensors.

\section{ASSOCIATED CONTENT}

\section{S Supporting Information}

The Supporting Information is available free of charge on the ACS Publications website at DOI: 10.1021/acssensors.7b00692.

Experimental description, HRSEM characterization and reflectivity spectra of the $\mathrm{PSiO}_{2}$ nanostructure, RIFTS characterization of aptamer immobilization, standard biosensing experiments with $7.5 \mu \mathrm{M}$ target concentration, ITP biosensing experiment with $7.5 \mathrm{nM}$ target, raw reflectivity data and corresponding FFT spectra of ITP biosensing experiments, ITP-based biosensing experiments without target protein, SDS-PAGE analysis of bacteria lysate samples, and sample separation and accumulation over time during ITP (PDF) 


\section{AUTHOR INFORMATION}

\section{Corresponding Author}

*E-mail: esegal@technion.ac.il.

ORCID

Moran Bercovici: 0000-0002-7803-4791

Ester Segal: 0000-0001-9472-754X

Notes

The authors declare no competing financial interest.

\section{ACKNOWLEDGMENTS}

This work was partially supported by the NEVET grant administrated by the Russell Berrie Nanotechnology Institute (RBNI), by the German Research Foundation under the grant SCHE 279/32-1 and by the Initial Training Network, Virtual Vials, funded by the FP7 Marie Curie Actions of the European Commission (FP7-PEOPLE-2013-ITN-607322). The oxidation process was performed at the Micro-Nano Fabrication Unit (MNFU), Technion. The authors thank Dr. Khaled Gommed for his assistance in preparation of the microfluidic channels and Prof. Yuval Shoham from the Department of Biotechnology and Food Engineering at the Technion for supplying the target protein. S.A.-G. is most grateful for the Miriam and Aaron Gutwirth scholarship and for TEVA Pharmaceutical Industries Excellence Scholarship for M.Sc. students in Analytical Chemistry.

\section{REFERENCES}

(1) Lin, V. S.-Y.; Motesharei, K.; Dancil, K.-P. S.; Sailor, M. J.; Ghadiri, M. R. A Porous Silicon-Based Optical Interferometric Biosensor. Science 1997, 278 (5339), 840-843.

(2) Jane, A.; Dronov, R.; Hodges, A.; Voelcker, N. H. Porous silicon biosensors on the advance. Trends Biotechnol. 2009, 27 (4), 230-239.

(3) Shtenberg, G.; Segal, E., Porous Silicon Optical Biosensors. In Handbook of Porous Silicon, Canham, L., Ed.; Springer International Publishing: Cham, 2014; pp 857-868.

(4) Kilian, K. A.; Bocking, T.; Gooding, J. J. The importance of surface chemistry in mesoporous materials: lessons from porous silicon biosensors. Chem. Commun. 2009, 6, 630-640.

(5) Weiss, S. M.; Rong, G.; Lawrie, J. L. Current status and outlook for silicon-based optical biosensors. Phys. E 2009, 41 (6), 1071-1075.

(6) Janshoff, A.; Dancil, K.-P. S.; Steinem, C.; Greiner, D. P.; Lin, V. S. Y.; Gurtner, C.; Motesharei, K.; Sailor, M. J.; Ghadiri, M. R. Macroporous p-Type Silicon Fabry-Perot Layers. Fabrication, Characterization, and Applications in Biosensing. J. Am. Chem. Soc. 1998, 120 (46), 12108-12116.

(7) Snow, P. A.; Squire, E. K.; Russell, P. S. J.; Canham, L. T. Vapor sensing using the optical properties of porous silicon Bragg mirrors. J. Appl. Phys. 1999, 86 (4), 1781-1784.

(8) Ilyas, S.; Böcking, T.; Kilian, K.; Reece, P. J.; Gooding, J.; Gaus, $\mathrm{K}$; Gal, M. Porous silicon based narrow line-width rugate filters. Opt. Mater. 2007, 29 (6), 619-622.

(9) Cunin, F.; Schmedake, T. A.; Link, J. R.; Li, Y. Y.; Koh, J.; Bhatia, S. N.; Sailor, M. J. Biomolecular screening with encoded porous-silicon photonic crystals. Nat. Mater. 2002, 1 (1), 39-41.

(10) Ouyang, H.; Christophersen, M.; Viard, R.; Miller, B. L.; Fauchet, P. M. Macroporous Silicon Microcavities for Macromolecule Detection. Adv. Funct. Mater. 2005, 15 (11), 1851-1859.

(11) Chan, S.; Fauchet, P.; Li, Y.; Rothberg, L.; Miller, B. Porous silicon microcavities for biosensing applications. physica status solidi (a) 2000, 182 (1), 541-546.

(12) Politi, J.; Dardano, P.; Caliò, A.; Iodice, M.; Rea, I.; De Stefano, L. Reversible sensing of heavy metal ions using lysine modified oligopeptides on porous silicon and gold. Sens. Actuators, B 2017, 244, $142-150$.
(13) Krismastuti, F. S. H.; Brooks, W. L. A.; Sweetman, M. J.; Sumerlin, B. S.; Voelcker, N. H. A photonic glucose biosensor for chronic wound prognostics. J. Mater. Chem. B 2014, 2 (25), 39723983.

(14) Bonanno, L. M.; DeLouise, L. A. Tunable Detection Sensitivity of Opiates in Urine via a Label-Free Porous Silicon Competitive Inhibition Immunosensor. Anal. Chem. 2010, 82 (2), 714.

(15) Bonanno, L. M.; Kwong, T. C.; DeLouise, L. A.; Label-Free, A. Porous Silicon Immunosensor for Broad Detection of Opiates in a Blind Clinical Study and Result Comparison to Commercial Analytical Chemistry Techniques. Anal. Chem. 2010, 82 (23), 9711-9718.

(16) Rong, G.; Ryckman, J. D.; Mernaugh, R. L.; Weiss, S. M. Labelfree porous silicon membrane waveguide for DNA sensing. Appl. Phys. Lett. 2008, 93 (16), 161109.

(17) De Stefano, L.; Arcari, P.; Lamberti, A.; Sanges, C.; Rotiroti, L.; Rea, I.; Rendina, I. DNA Optical Detection Based on Porous Silicon Technology: from Biosensors to Biochips. Sensors 2007, 7 (2), 214.

(18) Steinem, C.; Janshoff, A.; Lin, V. S. Y.; Völcker, N. H.; Reza Ghadiri, M. DNA hybridization-enhanced porous silicon corrosion: mechanistic investigations and prospect for optical interferometric biosensing. Tetrahedron 2004, 60 (49), 11259-11267.

(19) Urmann, K.; Reich, P.; Walter, J.-G.; Beckmann, D.; Segal, E.; Scheper, T. Rapid and label-free detection of protein a by aptamertethered porous silicon nanostructures. J. Biotechnol. 2017, 257, 171177.

(20) Urmann, K.; Walter, J.-G.; Scheper, T.; Segal, E. Label-Free Optical Biosensors Based on Aptamer-Functionalized Porous Silicon Scaffolds. Anal. Chem. 2015, 87 (3), 1999-2006.

(21) Gupta, B.; Mai, K.; Lowe, S. B.; Wakefield, D.; Di Girolamo, N.; Gaus, K.; Reece, P. J.; Gooding, J. J. Ultrasensitive and Specific Measurement of Protease Activity Using Functionalized Photonic Crystals. Anal. Chem. 2015, 87 (19), 9946-9953.

(22) Shtenberg, G.; Massad-Ivanir, N.; Moscovitz, O.; Engin, S.; Sharon, M.; Fruk, L.; Segal, E. Picking up the Pieces: A Generic Porous Si Biosensor for Probing the Proteolytic Products of Enzymes. Anal. Chem. 2013, 85 (3), 1951-1956.

(23) Massad-Ivanir, N.; Mirsky, Y.; Nahor, A.; Edrei, E.; BonannoYoung, L. M.; Ben Dov, N.; Sa'ar, A.; Segal, E. Trap and track: designing self-reporting porous $\mathrm{Si}$ photonic crystals for rapid bacteria detection. Analyst 2014, 139 (16), 3885-3894.

(24) Massad-Ivanir, N.; Shtenberg, G.; Tzur, A.; Krepker, M. A.; Segal, E. Engineering Nanostructured Porous $\mathrm{SiO} 2$ Surfaces for Bacteria Detection via "Direct Cell Capture. Anal. Chem. 2011, 83 (9), 3282-3289.

(25) Massad-Ivanir, N.; Shtenberg, G.; Zeidman, T.; Segal, E. Construction and Characterization of Porous $\mathrm{SiO} 2 /$ Hydrogel Hybrids as Optical Biosensors for Rapid Detection of Bacteria. Adv. Funct. Mater. 2010, 20 (14), 2269-2277.

(26) Urmann, K.; Arshavsky-Graham, S.; Walter, J. G.; Scheper, T.; Segal, E. Whole-cell detection of live lactobacillus acidophilus on aptamer-decorated porous silicon biosensors. Analyst 2016, 141 (18), 5432-5440.

(27) Pacholski, C.; Sartor, M.; Sailor, M. J.; Cunin, F.; Miskelly, G. M. Biosensing Using Porous Silicon Double-Layer Interferometers: Reflective Interferometric Fourier Transform Spectroscopy. J. Am. Chem. Soc. 2005, 127 (33), 11636-11645.

(28) Pacholski, C.; Yu, C.; Miskelly, G. M.; Godin, D.; Sailor, M. J. Reflective Interferometric Fourier Transform Spectroscopy: A SelfCompensating Label-Free Immunosensor Using Double-Layers of Porous SiO2. J. Am. Chem. Soc. 2006, 128 (13), 4250-4252.

(29) Sailor, M. J. Porous silicon in practice: preparation, characterization and applications; John Wiley \& Sons, 2012.

(30) Vilensky, R.; Bercovici, M.; Segal, E. Oxidized Porous Silicon Nanostructures Enabling Electrokinetic Transport for Enhanced DNA Detection. Adv. Funct. Mater. 2015, 25 (43), 6725-6732.

(31) Zhao, Y.; Gaur, G.; Retterer, S. T.; Laibinis, P. E.; Weiss, S. M. Flow-Through Porous Silicon Membranes for Real-Time Label-Free Biosensing. Anal. Chem. 2016, 88 (22), 10940-10948. 
(32) Lazzara, T. D.; Mey, I.; Steinem, C.; Janshoff, A. Benefits and Limitations of Porous Substrates as Biosensors for Protein Adsorption. Anal. Chem. 2011, 83 (14), 5624-5630.

(33) Rong, G.; Weiss, S. M. Biomolecule size-dependent sensitivity of porous silicon sensors. Phys. Status Solidi A 2009, 206 (6), 1365-1368.

(34) Rea, I.; Lamberti, A.; Rendina, I.; Coppola, G.; Gioffrè, M.; Iodice, M.; Casalino, M.; Tommasi, E. D.; Stefano, L. D. Fabrication and characterization of a porous silicon based microarray for label-free optical monitoring of biomolecular interactions. J. Appl. Phys. 2010, 107 (1), 014513.

(35) DeLouise, L. A.; Kou, P. M.; Miller, B. L. Cross-Correlation of Optical Microcavity Biosensor Response with Immobilized Enzyme Activity. Insights into Biosensor Sensitivity. Anal. Chem. 2005, 77 (10), $3222-3230$.

(36) Orosco, M. M.; Pacholski, C.; Miskelly, G. M.; Sailor, M. J. Protein-Coated Porous-Silicon Photonic Crystals for Amplified Optical Detection of Protease Activity. Adv. Mater. 2006, 18 (11), 1393-1396.

(37) Ouyang, H.; DeLouise, L. A.; Miller, B. L.; Fauchet, P. M. LabelFree Quantitative Detection of Protein Using Macroporous Silicon Photonic Bandgap Biosensors. Anal. Chem. 2007, 79 (4), 1502-1506.

(38) Mariani, S.; Strambini, L. M.; Barillaro, G. Femtomole Detection of Proteins Using a Label-Free Nanostructured Porous Silicon Interferometer for Perspective Ultrasensitive Biosensing. Anal. Chem. 2016, 88 (17), 8502-8509.

(39) Mariani, S.; Pino, L.; Strambini, L. M.; Tedeschi, L.; Barillaro, G. 10 000-Fold Improvement in Protein Detection Using Nanostructured Porous Silicon Interferometric Aptasensors. ACS Sensors 2016, 1 (12), 1471-1479.

(40) Karsenty, M.; Rubin, S.; Bercovici, M. Acceleration of SurfaceBased Hybridization Reactions Using Isotachophoretic Focusing. Anal. Chem. 2014, 86 (6), 3028-3036.

(41) Han, C. M.; Katilius, E.; Santiago, J. G. Increasing hybridization rate and sensitivity of DNA microarrays using isotachophoresis. Lab Chip 2014, 14 (16), 2958-2967.

(42) Moghadam, B. Y.; Connelly, K. T.; Posner, J. D. Two Orders of Magnitude Improvement in Detection Limit of Lateral Flow Assays Using Isotachophoresis. Anal. Chem. 2015, 87 (2), 1009-1017.

(43) Paratore, F.; Zeidman Kalman, T.; Rosenfeld, T.; Kaigala, G. V.; Bercovici, M. Isotachophoresis-Based Surface Immunoassay. Anal. Chem. 2017, 89 (14), 7373-7381.

(44) Malá, Z.; Gebauer, P.; Boček, P. Recent progress in analytical capillary isotachophoresis. Electrophoresis 2015, 36 (1), 2-14.

(45) Malá, Z.; Gebauer, P.; Boček, P. Analytical capillary isotachophoresis after 50 years of development: Recent progress 2014-2016. Electrophoresis 2017, 38 (1), 9-19.

(46) Jung, B.; Bharadwaj, R.; Santiago, J. G. On-chip millionfold sample stacking using transient isotachophoresis. Anal. Chem. 2006, 78 (7), 2319-27.

(47) Kökpinar, Ö.; Walter, J.-G.; Shoham, Y.; Stahl, F.; Scheper, T. Aptamer-based downstream processing of his-tagged proteins utilizing magnetic beads. Biotechnol. Bioeng. 2011, 108 (10), 2371-2379.

(48) Zhu, G.; Lübbecke, M.; Walter, J. G.; Stahl, F.; Scheper, T. Characterization of Optimal Aptamer-Microarray Binding Chemistry and Spacer Design. Chem. Eng. Technol. 2011, 34 (12), 2022-2028.

(49) Doyle, S. A.; Murphy, M. B. Aptamers and methods for their in vitro selection and uses thereof; Lawrence Berkeley National Laboratory (LBNL): Berkeley, CA (United States), 2008.

(50) Urmann, K.; Modrejewski, J.; Scheper, T.; Walter, J. G. Aptamer-modified nanomaterials: principles and applications. BioNanoMaterials 2017, 18, 1 DOI: 10.1515/bnm-2016-0012.

(51) Everaerts, F.; Beckers, J.; Verheggen, T. Isotachophoresis: Theory, Instrumentation and Practice; Elsevier: New York, 2011.

(52) Squires, T. M.; Messinger, R. J.; Manalis, S. R. Making it stick: convection, reaction and diffusion in surface-based biosensors. Nat. Biotechnol. 2008, 26 (4), 417-426.

(53) GanOr, N.; Rubin, S.; Bercovici, M. Diffusion dependent focusing regimes in peak mode counterflow isotachophoresis. Phys. Fluids 2015, 27 (7), 072003. 